\title{
System Identification and Modelling of a High Performance Hydraulic Actuator
}

\author{
Benoit Boulet, Laeeque Daneshmend, Vincent Hayward, Chafye Nemri \\ McGill Research Center for Intelligent Machines \\ McGill University \\ 3480 University Street, Montréal, Québec, CANADA H3A 2A7
}

\begin{abstract}
Detailed knowledge of actuator properties is a prerequisite for advanced manipulator design and control. This paper deals with the experimental identification and modelling of the nonlinear dynamics of a high performance hydraulic actuator. Such actuators are of interest for applications which require both high power and high bandwidth. An analytical model of the system is formulated, and a software simulator implementing the force-controlled actuator model including all the nonlinear elements is shown to predict the real system's behavior quite well. The actuator properties and performance are also discussed.
\end{abstract}

\section{Introduction}

Hydraulic actuation used to be, and in many cases, remains the technique of choice for high performance robotic applications. However, this type of actuation is not presently receiving a great deal of attention from the robotic research community despite its often ignored advantages. This may be due, in part, to unjustified prejudice against hydraulic systems on the part of robot designers in the research community.

Hydraulic actuation is often believed to be dirty, noisy, inaccurate, inadequate for force control, complicated to use, dangerous, expensive, and hard to package. These descriptions do indeed apply to certain, general purpose, hydraulic actuators. However, hydraulic actuators specifically designed for robotics and other demanding applications, such as those discussed in this paper, overcome many of these alleged shortcomings and offer a unique set of performance characteristics.

As the objectives in advanced manipulator research become increasingly demanding, the interaction among various components of the system, and the impact of this interaction on overall manipulator performance, becomes progressively more important. This necessitates an integrated approach to manipulator design: encompassing the kinematic, structural, actuation, sensing, and control aspects of the manipulator within a unified design process. Hence, detailed knowledge of actuator properties, and the nature of the 
limits on actuator performance, are a prerequisite for the integrated design of advanced manipulators. Actuator characteristics are of special relevance to control law design.

This paper focuses on the modelling and system identification of one particular high performance hydraulic actuator built by ASI. A physical model is derived for this actuator, and the parameters of the various components of this model are identified experimentally. The overall force loop performance of the actuator is also investigated, and compared to the predictions of a software simulator which implements the physical model.

\section{Actuator Overall Properties}

With proper design, leakage has been reduced to a minimum and can be easily controlled. In addition, modern quick release flexible supply lines make connecting and disconnecting a hydraulic unit almost as easy as connecting or disconnecting an electrical component. Due to lack of space, hydraulic supplies can only be discussed briefly here. These come in many designs, some of them very compact and convenient. In our case, we used an acoustically isolated conventional supply which is not noisier than say, a ventilated backplane chassis, and not more expensive than a bank of good quality DC motor amplifiers. The actuator itself is completely noise-free even at maximum thrust, that is $1340 \mathrm{~N}$ for $345 \mathrm{~N} / \mathrm{cm}^{2}$ (500 psi) supply. The turbulent flow is confined inside a solid metal manifold from which no audible (at least in our lab) acoustical noise can escape. This contrasts with some electro-mechanical equipment driven by switching power supplies. Also, the produced mechanical signal (force or velocity) is almost perfectly free of noise. This is typified by the sensation of smoothness when the controlled hydraulic actuator is made to interact with the experimenter's hand.

The device discussed here is a linear piston type actuator driven by an integrated highbandwidth jet pipe suspension valve, and fitted with a force sensor. It is very compact, mechanically robust, and its mass is about $.5 \mathrm{Kg}$ (17 ounces). A view of the actuator without the LVDT position sensor is shown at figure 1 . For a $76 \mathrm{~mm}$ stroke, the overall dimensions are $25 \times 55 \times 139 \mathrm{~mm}$. Since it is a force controlled device it must include some elasticity which is almost entirely lumped in the force sensor mounted directly on the cylinder. It thus may be considered as an active instrumented structural member easily integrated in a larger assembly.

The ASI servosystem also includes a controller card which can be accessed by a host computer. The card features on-board analog linear controllers whose gains can be programmed from a host computer, allowing gain scheduling. Digital control is also possible since the valve current can be specified as desired. The system state variables can be accessed either digitally via an on-board analog to digital converter or directly by measuring the analog signals.

Force control resolution is limited by the residual solid friction forces as seen at the piston rod in closed-loop operation. Thus, resolution depends on the ability of the internal driving force to overcome these forces, and by the resolution of the sensor itself. The closed-loop force feedback gain can be fairly high, hence the effects of residual friction can be made quite small. Consequently, sensor stiffness determines the basic tradeoff between force control bandwidth and resolution. These actuators must be essentially seen as force producers due to the four-way jet pipe design of its electromagnetic valve (single stage). 


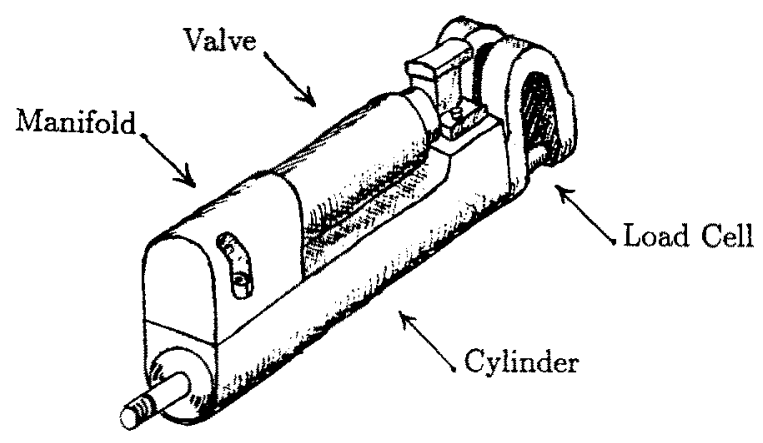

Figure 1: The AsI Hydraulic Actuator

The force output primarily results from the differential pressure across the lines leading to the chambers on each side of the piston. The pressure imbalance due to the suspension deviation is the fundamental operational mechanism. Because the valve is piggy-backed on the piston, a very direct connection between suspension deviation and force output is established.

Among the several major nonlinear characteristics of this actuator, hydraulic damping has a notable effect on performance. Hydraulic damping is a force which opposes the piston motions due to the circulation of oil through the valve orifices. For a fixed valve current that specifies a certain valve position, the effect is very small at low velocities, which make it difficult to assess, but increases faster than linearly for a certain velocity range, past which the characteristic curve tapers off. We conjecture that this effect is attributed to flow forces which become significant enough to force the opening of the valve. This phenomenon happens only when an external force applied on the piston adds up to the fluid pressure to produce higher velocities (and thus higher flow rates) than usually obtained. Thus, the force response bandwidth, kept at a maximum for small amplitude motions such as constrained or contact motions, is drastically decreased for fast motions of an inertial load in free space because the resultant velocities are in the range where the damping is exponential, enhancing stability. Hence the actuator has the intrinsic property to adapt its natural impedance characteristic to the type of tasks required in robotics. At the limit, when the fully opened valve forces maximum flow in and out the chambers, velocity saturates and is maintained constant for large variations of the disturbing load forces, as the thrust force would augment rapidly should the velocity drop. At the other end of the spectrum, when the velocity is small, the suspension deviation has a direct impact on the force output, resulting in high bandwidth force control.

High reliability is facilitated by a very small number of parts of which only two are moving parts: the bending jet pipe and the piston, not counting the LVDT position sensor. Solid friction only occurs between the piston, the rod and the cylinder in the entire assembly. The force sensor has inherent mechanical overload protection which enhances further reliability. Furthermore, elastic displacements are sensed by a noncontact Halleffect transducer. Finally the actuator can reach its mechanical travel limit at full valve opening without incurring any damage as the oil, forced out of the vanishing chamber 
volume, smoothly damps the motion to a stop. In these conditions, no external mechanical stops are required since they are built-in the actuator and can be adjusted to any requirement.

In summary, this actuator may be characterized as a direct drive device since the power derived from the input fuid pressure is almost directly applied to the load without any need for a motion transmission mechanism, with the valve acting as a variable gain amplifying element. It can thus be conceptually compared to an operational amplifier producing the best of its performance when linearized by high feedback gains.

In the coming sections, we shall dwell in some detail into the modelling of this device with a view to its use for force control.

\section{$3 \quad$ Physical Modelling}

A "gray-box" model approach was adopted since a number of the system parameters were not known and in most cases were unavailable information. Some "reverse engineering" was performed to develop an understanding of how the system elements were designed. Our model includes linear dynamics in conjuction with nonlinear elements. These are hysteresis, static valve force characteristic, hydraulic damping and friction. These nonlinearities play an important role in the actual system and must be included if the model's predictions are to be a good approximation of the actuator's behavior. A block diagram of the closed-loop model is presented at figure 2. The linear blocks represent the valve, fluid, and force sensor dynamics, which are respectively denoted as $G(z), D(z)$ and $S(s)$. Zero-order holds are used at the outputs of the discrete-time blocks but are not shown on the figure.

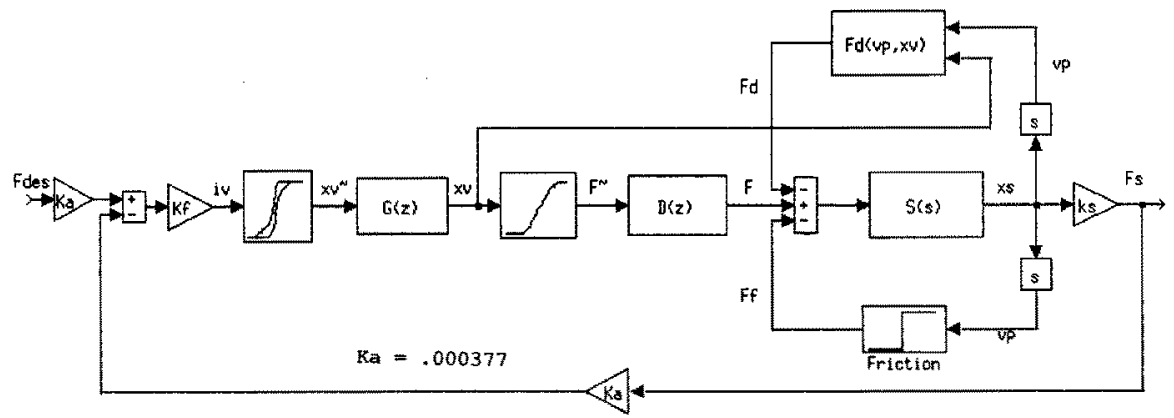

Figure 2: Block Diagram of the Closed-Loop Model

The supply pressure $\left(345 \mathrm{~N} / \mathrm{cm}^{2}\right)$ was the only available a priori information before ASI kindly agreed to provide us with proprietary information regarding the geometry of the valve. This information was needed to calculate the valve force versus the valve pipe tip position static characteristic $\tilde{F}\left(x_{v}\right)$ i.e. the static hydraulic force applied on the piston when it is constrained to a null velocity. All the other system parameters were unknown and had to be measured or identified. 
The unknown, but measurable, model parameters were the sensor calibration, the force sensor dynamics and stiffness, the valve hysteresis characteristic, the friction characteristic and the hydraulic damping effect. The unknown, but identifiable, model parameters were the valve and fluid dynamics.

\subsection{Valve Static Force Characteristic}

A mathematical model of the valve static force characteristic $\tilde{F}\left(x_{v}\right)$ was worked out. Two assumptions were made. Firstly, the flow through the valve orifices was assumed to vary with the square root of the pressure difference across the orifices. Orifice discharge coefficients could not be measured and were estimated, based on values given in [2] (pp. 181-183). The second assumption was that direct leakage from the valve pipe tip to the return chamber is negligible. This is justified considering that even if there is some leakage, its effect should be mostly independent of $x_{v}$ and should roughly be equivalent to a drop in pressure at the end of the supply line, thereby affecting only the saturation force values but not the general shape of the function. An expression was derived to calculate the steady-state force with respect to the valve pipe tip position and its characteristic is shown at figure 3. Each saturation force corresponds to the area on each side of the piston multiplied by the supply pressure.

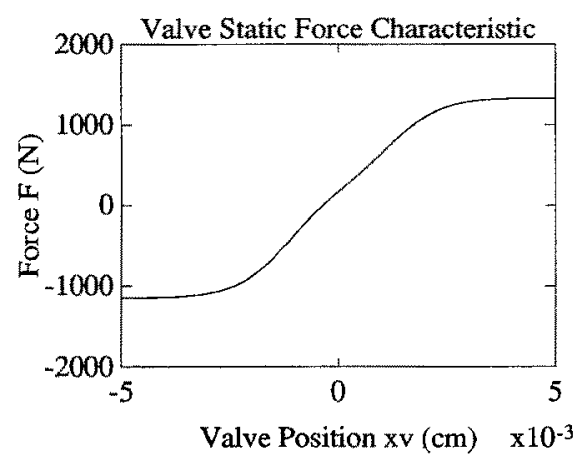

Figure 3: Valve Static Force Characteristic

It is seen that the output force is not null but has a positive bias when the valve position is zero. This is the case if the valve pipe tip position is measured from a geometrically centered origin, but an offset in valve current can be set to approximately compensate any mechanical bias such that the output force is about zero. This has the effect of shifting the valve static force characteristic and it is taken care of in the model by adding a negative offset to $x_{v}$ before the function simulating the force characteristic is called.

\subsection{Sensor Calibration and Dynamics}

Calibration of the position sensor is performed by adjusting an offset and a gain and by measuring the piston stroke. The force sensor is calibrated similarly but its stiffness has 
solid contact, thus a mass-spring-damper second-order dynamic model was chosen. We assumed that the fixture used for experimentation was perfectly rigid, although actual results showed significant bending.

\subsection{Valve Hysteresis}

Valve hysteresis is significant and a model was built to account for it. The model is based on a technique described in [4]. It is capable of generating minor loops from the knowledge of the major hysteresis loop. In the model, the input to the hysteresis block is the valve current $i_{y}$ and the output is the DC valve pipe tip position $\tilde{x}_{y}$. Hysteresis output is usually chosen to be the valve's motor torque but we couldn't measure it. Hence, although the relationship between $i_{v}$ and $x_{v}$ would normally include the valve dynamics, we had to separate the DC hysteresis characteristic from the dynamics which relate the static and actual valve positions, $X_{v}(z) / \tilde{X}_{v}(z)=G(z)$.

The valve hysteresis is included in the system static force characteristic which can easily be measured. Friction is also included in the static force characteristic but we neglected it because of uncertainty in our friction model at very low velocities. We used the inverse of the calculated nonlinear valve static force function to obtain the lower and higher parts of the $\tilde{x}_{v}\left(i_{v}\right)$ hysteresis major loop from the DC characteristic data.

\subsection{Friction Model}

The friction model includes kinetic friction only. Numerical oscillation problems were avoided in the simulator by using a modified Dahl model (see [10]). The expression of the time-derivative of the friction force is:

$$
\begin{aligned}
\partial F_{f} / \partial t & =\gamma\left(F_{f}-F_{c} \operatorname{sgn}\left(v_{p}\right)\right)^{2} v_{p}, \\
\text { where } \gamma & =100, \\
F_{c} & =26 \mathrm{~N} \quad \text { (Coulomb Friction). }
\end{aligned}
$$

The parameter $\gamma$ in equation (1) is set to a suitable value for a fast transient in $F_{f}$ towards the Coulomb friction $F_{c}$ or $-F_{c}$ when $v_{p}$ changes sign. It should be noted that the use of this friction model which, in steady-state, is equivalent to a simple Coulomb friction model, was only intended for improving the numerical integration and not for modelling the actual Dahl effect.

\subsection{Hydraulic Damping Effect}

The hydraulic damping force depends on the valve pipe tip position and on the piston velocity. The family of curves used to model this effect is based on experimental data and thus it includes the flow forces acting on the valve pipe tip. Although the valve position can't be measured, we used the knowledge of the desired input currents and found the corresponding valve positions by applying these current values to the hysteresis model. The flow forces on the valve pipe and the uncertainty in the hysteresis model limit our ability to accurately predict the valve position. 


\subsection{Identification of Valve and Fluid Dynamics}

The valve and fluid dynamics had to be identified for parametrization of the linear blocks in the model. All the linear dynamics were identified as a whole and several assumptions were made in order to be able to select the right poles and zeros for each transfer function.

It was assumed that the valve was the most restrictive limit to the open-loop bandwidth and this was based on the figures used for a similar valve in [7]. A second-order model with two distinct real poles was expected to give good results because of severe damping applied on the valve pipe tip by the fluid in the return chamber.

For the fluid dynamics, the supply and return lines were assumed to be lumpedparameter linear second-order systems. The parameters are the fluid inertia, the fluid and line compliance and the orifice resistance. The chambers on each side of the piston were assumed to be lumped-parameter first-order linear systems, the parameters being the fluid compliance and the orifice resistance. The overall fluid dynamic model order is six.

Two poles should be related to the force sensor dynamics in the identified linear transfer function which should be of the tenth order. These poles were expected to be complex and located below the force sensor's natural frequency because of the hydraulic damping effect, which is assumed to be small since the PRBS input used for identification had a low amplitude.

\subsection{Actuator Model}

A diagram of the physical actuator model is presented at figure 4 . The dynamic and output equations relating the hydraulic force $F$ to the sensed force $F_{s}$ are:

$$
\begin{aligned}
F\left(x_{v}\right)-F_{d}\left(x_{v}, v_{p}\right)-F_{f}\left(v_{p}\right) & =m \ddot{x}_{s}+b_{s} \dot{x}_{s}+k_{s} x_{s} \\
F_{s} & =k_{s} x_{s} .
\end{aligned}
$$

where:

$$
\begin{array}{llll}
F_{d}\left(x_{v}, v_{p}\right) & \equiv \text { hydraulic damping force } & x_{s} & \equiv \text { force sensor deflection } \\
F_{f}\left(v_{p}\right) & \equiv \text { friction force } & m & \equiv \text { actuator mass minus piston mass } \\
v_{p} & \equiv \text { piston velocity } & b_{s}, k_{s} & \equiv \text { force sensor parameters }
\end{array}
$$

\section{Experimentation}

\subsection{Measurement of Force Sensor Characteristics}

As a first experiment, we had to measure the force sensor characteristics. We directly measured the force sensor stiffness by locking the piston to the mount and by measuring the total sensor deflection as full output force was applied in both directions. Then, by using the known saturation force values, we were able to calculate the sensor stiffness. One disadvantage of this method is that full sensor deflection probably covers a nonlinear domain of the sensed force $F_{s}$ vs sensor position $x_{s}$ relationship.

The force sensor impulse response was also measured by gently knocking the actuator with a piece of metal while it was held vertical. The damping factor $\zeta$, natural frequency 


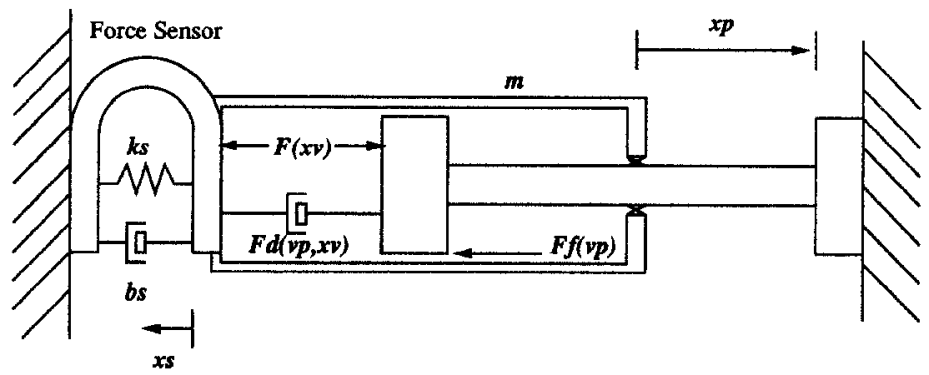

Figure 4: Actuator Model

$\omega_{n}$, damping coefficient $b_{s}$ and sensor stiffness $k_{s}$ were then calculated and are presented at table 1. It was noted that the impulse response gave much better results than the step response because in the latter case, lateral modes were excited and masked the effect of the desired axial mode. The sensor stiffness value used in the model for simulation is the one derived from the impulse response experiment. Equation (4) is the force sensor transfer function $S(s)$ used in the model.

$$
S(s)=\frac{178.6}{s^{2}+758.4 s+6712857}
$$

\begin{tabular}{|c|c|c|}
\hline \multicolumn{2}{|l|}{ actuator mass $m_{a}$} & $0.612 \mathrm{~kg}$ \\
\hline \multicolumn{2}{|c|}{ actuator mass minus piston mass $m$} & $\approx 0.560 \mathrm{~kg}$ \\
\hline \multirow[t]{2}{*}{ sensor stiffness $k_{s}$} & (direct) & $43659 \mathrm{~N} / \mathrm{cm}$ \\
\hline & (impulse) & $37592 \mathrm{~N} / \mathrm{cm}$ \\
\hline natural frequency $\omega_{n}=\sqrt{k_{s} / m_{a}}$ & & $2478 \mathrm{rad} / \mathrm{s}(394 \mathrm{~Hz})$ \\
\hline damping factor $\zeta$ & & 0.14 \\
\hline viscous damping coefficient $b_{s}$ & & $4.25 \mathrm{~N} / \mathrm{cm} / \mathrm{s}$ \\
\hline
\end{tabular}

Table 1: Measured Force Sensor Parameters

\subsection{Measurement of Open-Loop Static Force Characteristic}

With the piston locked to the mount, the open-loop static force characteristic was recorded while the valve current was slowly varied step by step following a triangular input. The current driver sensitivity allowed $.488 \mathrm{~mA}$ increments in valve current. The static force characteristic and the calculated hysteresis major loop are shown at figure 5 .

\subsection{Measurement of Friction}

Kinetic friction and stiction were measured after the oil had been taken out of the actuator (some oil was left, providing lubrication). The main disadvantage of this method is that 


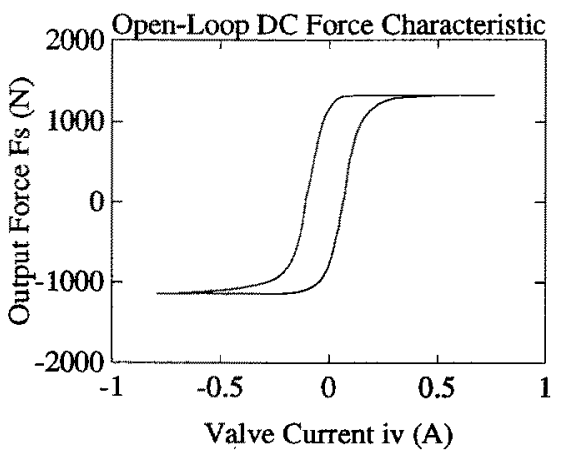

(a)

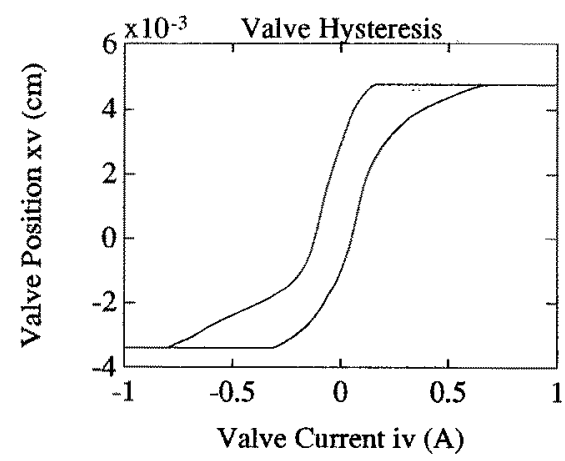

(1)

Figure 5: (a) Open-Loop Static Force Characteristic, (b) Valve Hysteresis

friction is likely to change when the pressure across the piston varies as the seal gets squeezed. In situ differential pressure measurements would give more accurate assessment of the phenomenon. Stiction was measured as the force at the breaking point where the piston starts moving. Coulomb friction was measured by pulling on the piston by hand and recording the force and the piston velocity. Results are shown at table 2 .

\begin{tabular}{|ll|r|}
\hline stiction & (pushing on piston) & $99 \mathrm{~N}$ \\
\cline { 2 - 3 } & (pulling on piston) & $-54 \mathrm{~N}$ \\
\hline Coulomb friction & $\approx 26 \mathrm{~N}$ \\
\hline
\end{tabular}

Table 2: Friction Measurements

\subsection{Measurement of Damping Effect}

For the hydraulic damping experiment, the actuator was mounted vertically such that weights could be hung from the piston (which was free to move). The procedure was as follows: we used a certain valve current as input to the open-loop system and measured the piston steady-state velocity without any load hanging to it. The corresponding steadystate force applied by the fluid pressure on the piston couldn't be measured directly but was found later by locking the piston and measuring the output force for the same input current. It is important to note that the sequence of applied currents must be the same for the hysteresis to operate in the same region. Then, for the same input current, different masses were hung to the piston and the corresponding steady-state velocities were measured. For each of these masses, the total force applied on the piston could be calculated as the sum of the measured hydraulic force and the gravitational force acting on the mass. It was assumed that the hydraulic reaction force was equal to that sum, i.e. we neglected the friction force. This procedure, which provided experimental data for one value of valve current, was repeated for different valve currents in order to be able to fit a family of curves to the data. 
A family of hyperbolic tangents whose magnitudes, scalings and positions with respect to the origin depend on the valve position $x_{v}$ has been fitted to the experimental data (see equation (5) below). Cubic splines were used for interpolation between the experimental values of $A\left(x_{v}\right), s\left(x_{v}\right)$ and $d\left(x_{v}\right)$. A linear damping term was added. The curves fitted to the experimental data are shown at figure 6.

It is interesting to note how the incremental hydraulic damping force decreases as $v_{p}$ increases past a certain value depending on the valve position, whereas the damping force was expected to follow the usual small orifice square relationship between the flow and the pressure. As stated earlier on, this is probably due to the flow forces acting on the valve which would tend to open it as the piston velocity increases, thus causing the incremental force to get smaller.

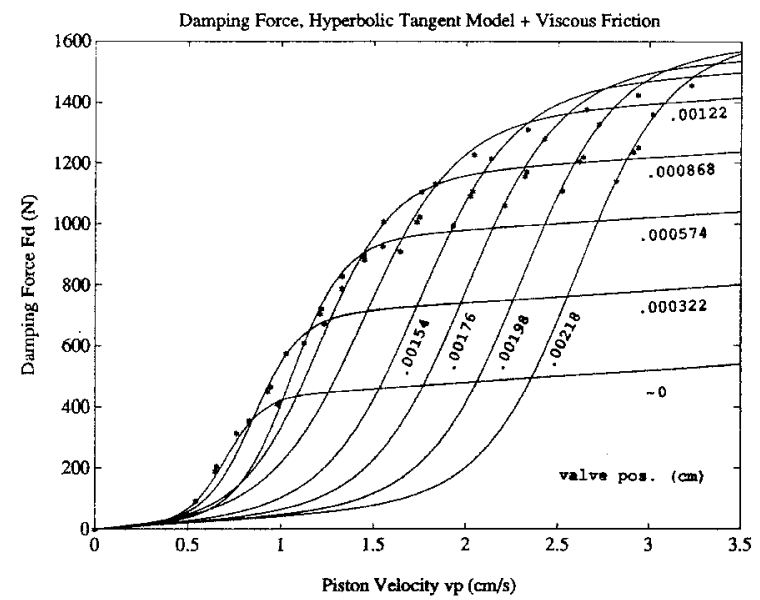

Figure 6: Experimental Hydraulic Damping Effect

$$
\begin{aligned}
F_{d} & =A\left(\tanh \left(s\left(\left|v_{p}\right|+d\right)\right)-\tanh (s d)\right) \operatorname{sgn}\left(v_{p}\right)+40 v_{p}, \\
\text { where } \quad A & =A\left(x_{v}\right), s=s\left(x_{v}\right), \quad d=d\left(x_{v}\right)
\end{aligned}
$$

The first simulations showed that the model had too much damping and a gain of .4 was added at the output of the hydraulic damping block. The model also uses bandlimited differentiation (a pole added at $40 \mathrm{~Hz}$ ) to reduce numerical noise problems arising in the nonlinear damping loop and to improve the closed-loop response.

\subsection{Identification of the Linear Part}

Separate identification of the valve dynamics and the fluid dynamics was not possible; we had to identify the linear part as a whole. With the piston fixed at midstroke position, a low-amplitude PRBS input was applied to the open-loop system so that we could assume that the system was operating in a linear region. It should be noted that the fluid compliance in the cylinder depends on the piston position and reaches a maximum when 
the piston is at a point where both chamber volumes are equal. Therefore, the case studied here was nearly the most adverse condition to stable control when considering only the fluid dynamics (see [11], pp. 50-51). The sampling frequency was $5000 \mathrm{~Hz}$.

An ARX model was estimated using a least-squares method on MATLAB ${ }^{T M}$ (ARX command) and the best fit was given by a tenth-order model with two delays as predicted:

$$
\left(1+a_{1} z^{-1}+a_{2} z^{-2}+\cdots+a_{10} z^{-10}\right) Y(z)=\left(b_{3} z^{-3}+b_{4} z^{-4}+\cdots+b_{10} z^{-10}\right) U(z),
$$

$$
\text { where } \begin{aligned}
a_{1} & =-0.4223, a_{2}=-0.3765, a_{3}=-0.2802, a_{4}=-0.1959, a_{5}=0.1930, \\
& a_{6}=0.2234, a_{7}=-0.0532, a_{8}=-0.0051, a_{9}=-0.0903, a_{10}=0.0394, \\
& b_{3}=0.0997, b_{4}=-0.1360, b_{5}=-0.0258, b_{6}=0.1094, b_{7}=-0.4047, \\
b_{8} & =-0.1323, b_{9}=-0.4253, b_{10}=-0.7851 .
\end{aligned}
$$

The PRBS input and the system and ARX model outputs are shown respectively at figures 7 (a) and (b).The pole-zero plot of the identified model is shown in Figure 8: as can be seen, the zeros of the identified model lie outside the unit circle. This indicates that the system identification technique has yielded a non-minimum phase model. The physical system has several components which are actually distributed parameter systems, e.g. hydraulic fluid and lines, valve stem flexure, etc., and there also exist possibilities of multiple transmission paths due to the mechanics of the test set-up. Hence the nonminimum phase nature of the model appears justified. Fortunately, these non-minimum phase zeros are clustered at high frequencies. Hence controller design can be based upon frequency separation, by using an additional compensator block which filters out the high frequency behavior.
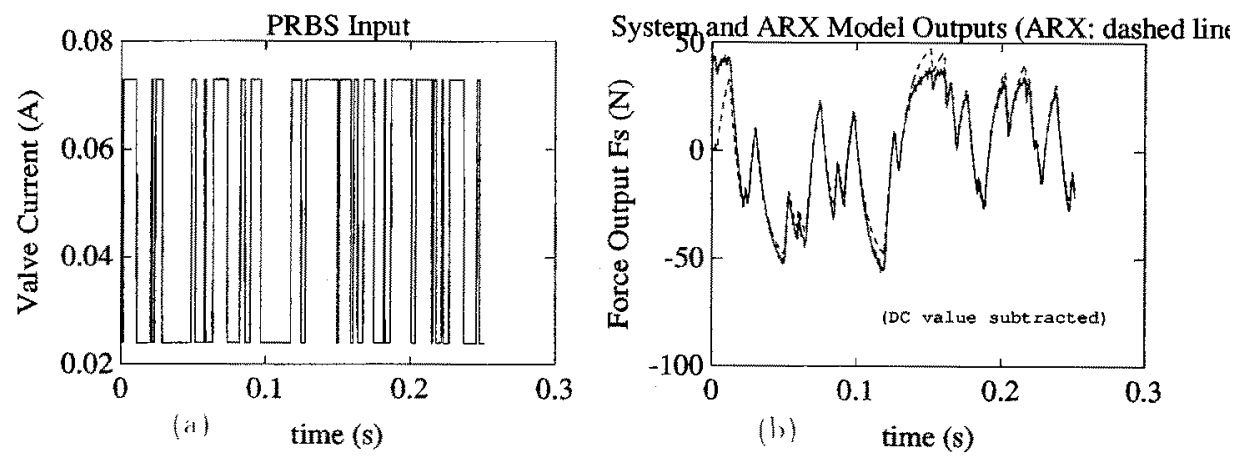

Figure 7: (a) PRBS Input, (b) System and ARX Model Outputs

\subsection{Dynamics}

As it was pointed out earlier on, the valve dynamics should have the lowest bandwidth and we therefore picked the only two identified real poles plus a zero at $z=0$ for $G(z)$ :

$$
G(z)=\frac{0.01203 z}{(z-0.9762)(z-0.4947)}, \quad|z|>0.9762
$$




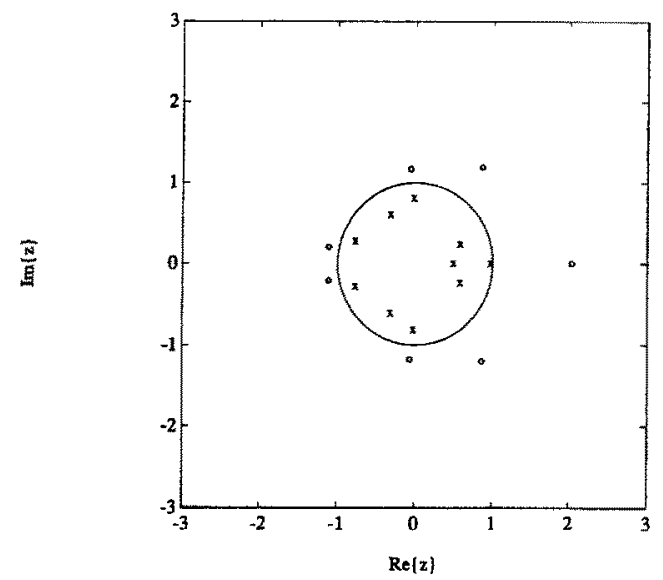

Figure 8: Poles and Zeros of Identified Transfer Function.

For the fluid dynamics, we picked the three pairs of complex poles at high frequencies and the three pairs of complex non-minimum phase zeros. We also chose the only real zero at $z=2.0282$ and placed two poles at $z=0$ to make $D(z)$ causal (see equation (8)). These two poles get cancelled with the zero of $G(z)$ and a zero at $z=0$ attributed to the force sensor dynamics in the identified transfer function. For the sensor dynamics, a pair of complex poles ${ }^{1}$ around $300 \mathrm{~Hz}$ and one zero at $z=0$ were disregarded.

$$
\begin{aligned}
D(z) & =\frac{-.67(z-2.03)\left(z^{2}-1.71 z+2.16\right)\left(z^{2}+0.14 z+1.39\right)\left(z^{2}+2.24 z+1.30\right)}{z^{2}\left(z^{2}+1.546 z+0.671\right)\left(z^{2}+0.036 z+0.657\right)\left(z^{2}+0.631 z+0.469\right)},(8) \\
|z| & >0.8191
\end{aligned}
$$

\subsection{Open-Loop and Closed-Loop Force Bandwidth}

The open-loop force bandwidth has been measured with the piston locked to the mount, and was found to be around $20 \mathrm{~Hz}$, a figure comparable to the achievable bandwidth of high-performance electric motors with current force sensors. It was noted that open-loop control was impractical because of the presence of hysteresis and friction.

Assuming that the force closed-loop system is linear for a given amplitude of the sinusoidal input, frequency responses were experimentally obtained and are shown at figures 9 (a) and (b) for different amplitudes of the input and for a force feedback gain of 2.44. The roll-off on the magnitude Bode plot (figure 9 (a)) indicates that the system is at least of the ninth order. The closed-loop bandwidth is around $100 \mathrm{~Hz}$ and decreases for higher input amplitudes. This is due in part to saturating nonlinear elements in the system but also to the nonlinear hydraulic damping. The $6 \mathrm{~dB}$ bandwidth goes as high as $196 \mathrm{~Hz}$ for low-amplitude inputs.

It should be noted that an on-board lag compensator can be added so that the proportional feedback gain can be lowered to get less overshoot without compromising the

${ }^{1}$ these poles are at $z=0.5797+0.2408 j$ and $z=0.5797-0.2408 j$ 


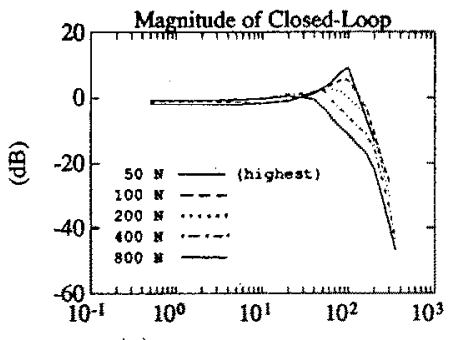

(a) freq. (Hz)

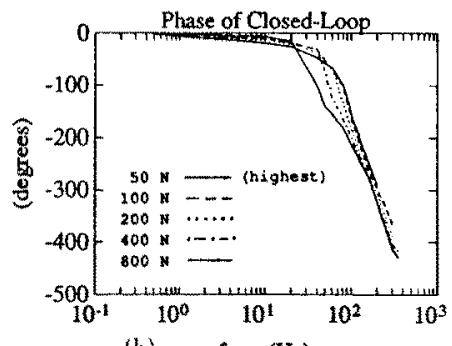

(b) freq. (Hz)

Figure 9: Closed-Loop Frequency Responses: (a) Magnitude, (b) Phase $\left(K_{f}=2.44\right)$

precision at low frequencies. A limit cycle has been observed for gain values of 3.66 and higher (sustained oscillations at frequencies around $95 \mathrm{~Hz}$ ). It was also observed that the closed-loop responses to sinusoid inputs (see figures 10 (a) and (b)) present little distortion given the degree of nonlinearity of the system. The slight distortions seen for high-amplitude, low-frequency responses (e.g. figure $10(\mathrm{~b})$ ) are probably due to the piece of aluminum on which the actuator was mounted: the assembly was such that this part of the fixture bent significantly for high output forces. The fixture also had an asymmetric, nonlinear, stiffness characteristic, so that it absorbed some elastic energy from the system and then suddenly released it as it moved back and forth. This could be observed for open-loop responses as well. Another explanation would be that the flow forces acting on the valve pipe tip would slightly disturb its position, thus causing a distortion in the output force. A better experimental rig is being constructed for future experimentation.
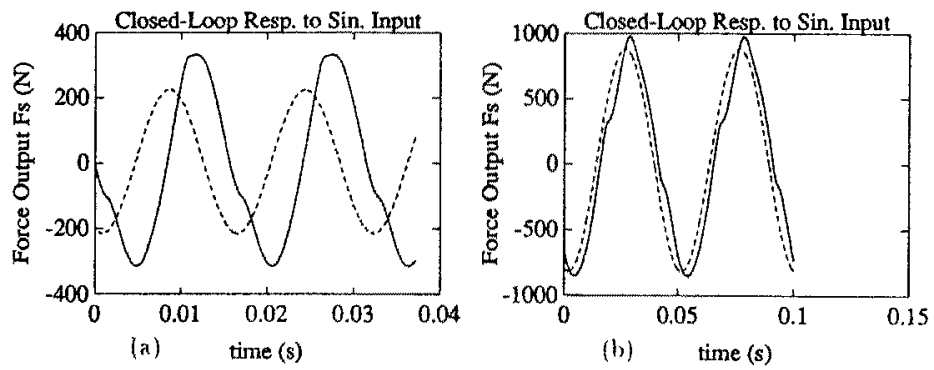

Figure 10: Closed-Loop Force Responses: (a) $f=63 \mathrm{~Hz}$, (b) $f=20 \mathrm{~Hz}$

\section{Simulation Results}

A software simulator has been built using SIMULAB ${ }^{T M}$. The simulator includes discretetime and continuous-time linear transfer functions. Zero-order holds are used at the output of the discrete-time blocks. The fifth-order Runge-Kutta integration algorithm was chosen for the simulations. 
The experimental and model closed-loop force responses (with feedback gain $K_{f}=2.44$ ) to a square-wave input agree fairly well for amplitudes of $200 \mathrm{~N}$ and $800 \mathrm{~N}$ (see figures 11 and 12). The $50 \mathrm{~N}$ model response is overdamped when compared to the experimental response (see figure 13). This is not surprising since the level of uncertainty in the combined effects of hydraulic damping and friction lies in the range of the $50 \mathrm{~N}$ response. The simulations show that the hydraulic damping and friction models are not really satisfactory at low velocities and low pressures. The kinetic friction might be less than expected for low pressures across the piston as it could explain why the simulated $50 \mathrm{~N}$ response is overdamped. Another potential source of error comes from the fact that the hysteresis model can't casily reproduce the small minor loops. The lower and higher parts used to construct the major loop were experimentally obtained and although some filtering was done on them, they are not locally perfectly smooth. Moreover, using the inverse of the valve static force function amplified these imperfections.

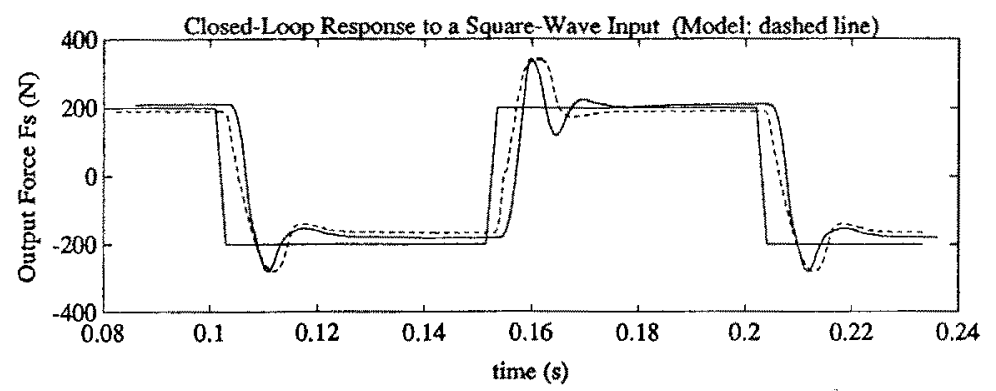

Figure 11: Closed-Loop Force Response to a $200 \mathrm{~N}$ Square-Wave Input $\left(K_{f}=2.44\right)$

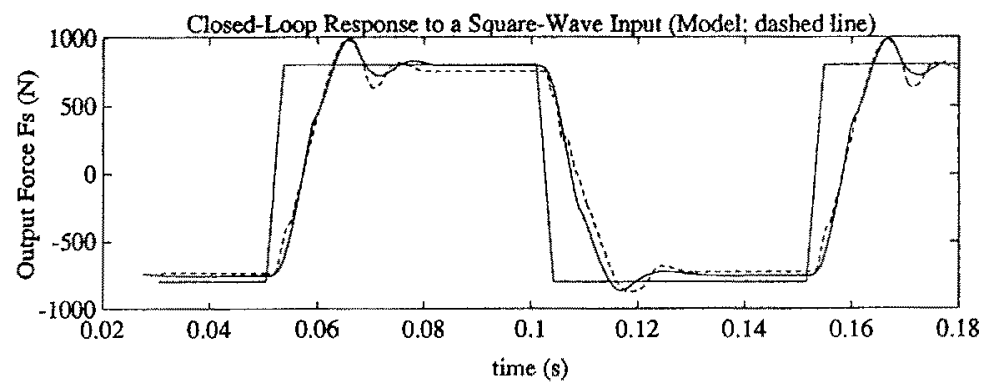

Figure 12: Closed-Loop Force Response to a $800 \mathrm{~N}$ Square-Wave Input $\left(K_{f}=2.44\right)$

\section{Conclusion}

A complete nonlinear model of the high-performance AsI hydraulic servosystem has been obtained, validated and simulated. The model's ability to reproduce experimental closedloop force responses for different amplitudes indicates that it could be a valuable tool for 


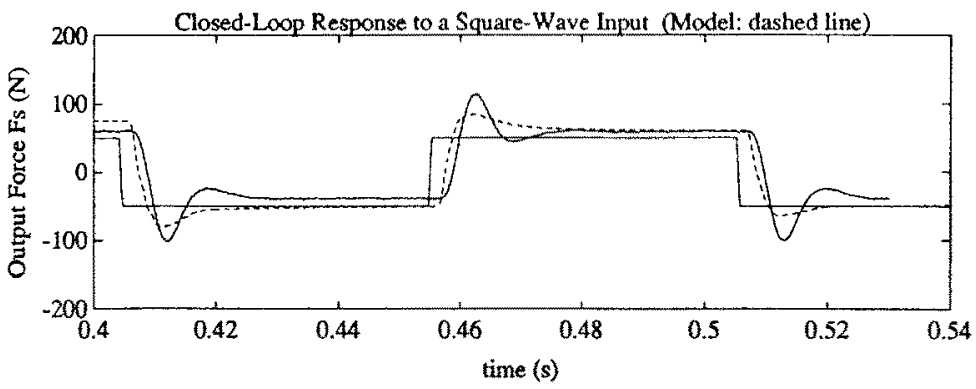

Figure 13: Closed-Loop Force Response to a $50 \mathrm{~N}$ Square-Wave Input $\left(K_{f}=2.44\right)$

the design of better digital nonlinear force control laws. It could also be useful to explain the sytem's behavior. The model can easily be extended for simulation of position and impedance control.

The open-loop force bandwidth $(20 \mathrm{~Hz})$ of the hydraulic actuator is comparable to the achievable force bandwidth of high-performance electric motors while the closed-loop force bandwidth was shown to be roughly $100 \mathrm{~Hz}$ for $K_{f}=2.44-$ much higher than any reported electric motor coupled to a force sensor of similar compliance. It must be remembered that many of the parameters reported in this paper are not intrisic to the actuator and can be modified to tradeoff various performance criteria. One of these parameters is sensor stiffness which is directly related to force control bandwidth.

The implications of the frequency response of the hydraulic actuator for control law design are encouraging: it is predominantly low-pass and the non-minimum phase zeros are clustered at high frequencies. Based on the dynamics, together with the large gains possible due to the high saturation level of the actuator, it appears ideally suited to feedback modulation of impedance over a wide range.

Further investigation into the identification of the linear dynamics is required to ascertain whether the non-minimum phase zeros in the model are artefacts of the system identification technique, or whether they can be related to specific distributed-parameter components of the overall system. Since non-minimum phase zeros place absolute limits on the achievable sensitivity minimization using feedback control, establishing the physical meaning of these zeros would be of relevance in further refining the actuator design to achieve even higher performance.

Better experiments will have to be designed for more satisfying models of the hydraulic damping and friction characteristics. The hydraulic actuator model will be used to assess the attainable range of mechanical impedance, very important for the study of antagonistic actuation. This type of actuation is required by a novel type of manipulator under construction at McGill. Some of its design features are discussed in [6]. It would be desirable to see the effect of reducing the model order by neglecting some of the fluid high frequency dynamics, and to explore digital nonlinear force and impedance control as well as dither. The system's ability to act as a force regulator while the piston is moving will also have to be assessed. 


\section{Acknowledgments}

The authors wish to acknowledge support from the Institute for Robotics and Intelligent Systems (IRIS) of Canada's Centers of Excellence Program (projects C-2 and C-3). Funding from NSERC the National Science and Engincering Council and FCAR Les Fonds pour la Formation des Chercheurs et l'Aide à la Recherche, Québec is also gratefully acknowledged.

Assistance from the staff with the Center for Engineering Design at the University of Utah and with ASI was fully appreciated. Skillful and diligent help from John Foldvari has been essential to carry out the experiments.

\section{References}

[1] Animate Systems Incorporated (ASI) 1991. Advanced Robotic Controller System Manual. Salt Lake City, Utah.

[2] Blackburn, J. F., Reethof, G., Shearer, J. L. (Eds.) 1960. Fluid Power Control. Cambridge: The MIT Press.

[3] Bobrow, J. E., Desai, J. 1990. Modeling and Analysis of a High-Torque, Hydrostatic Actuator for Robotic Applications. Experimental Robotics I, the First Int. Symp. V. Hayward, O. Khatib (Eds.) Springer-Verlag, pp. 215-228.

[4] Frame, J. G., Mohan, N., Liu, T. 1982. Hysteresis Modeling in an Electromagnetic Transients Program. IEEE Trans. on Power Apparatus and Systems Vol. PAs-101, No. 9, September, pp. 3403-3411.

[5] Gille, J. C., Decaulne, P., Pélegrin, M. 1985. Dynamique de la commande linéaire. Paris: Dunod. (in french)

[6] Hayward, V. 1991. Borrowing Some Design Ideas From Biological Manipulators to Design an Artificial One. Robots and Biological Systems, NATO Advanced Research Workshop. Dario P., Sandini, G., Aebisher, P. (Eds.), Springer-Verlag, in press.

[7] McLain, T. W., Iversen, E. K., Davis, C. C., Jacobsen, S. C. 1989. Development, Simulation, and Validation of a Highly Nonlinear Hydraulic Servosystem Model. Proc. of the 1989 American Control Conference, AACC. Piscataway: IEEE.

[8] Shearer, J. L. 1983. Digital Simulation of a Coulomb-Damped Hydraulic Servosystem. Trans. ASME, J. Dyn. Sys., Meas., Contr. Vol. 105, December, pp. 215-221.

[9] The Math Works Inc. 1990. sIMULAB: A Program for Simulating Dynamic Systems. (user's guide)

[10] Threlfall, D. C. 1978. The Inclusion of Coulomb Friction in Mechanisms Programs with Particular Reference to DRAM. Mech. and Mach. Theory Vol. 13, pp. 475-483.

[11] Walters, R. 1967. Hydraulic and Electro-Hydraulic Servo Systems. Cleveland: CRC Press. 